\title{
Article \\ Complete Mitogenomes of Two Aragoa Species and Phylogeny of Plantagineae (Plantaginaceae, Lamiales) Using Mitochondrial Genes and the Nuclear Ribosomal RNA Repeat
}

\author{
Jeffrey P. Mower ${ }^{1,2, *}$, Lilly Hanley ${ }^{1}$, Kirsten Wolff ${ }^{3}$, Natalia Pabón-Mora ${ }^{4}$ and Favio González ${ }^{5}$ \\ 1 Center for Plant Science Innovation, University of Nebraska, Lincoln, NE 68588, USA; noemail@noemail.edu \\ 2 Department of Agronomy and Horticulture, University of Nebraska, Lincoln, NE 68583, USA \\ 3 School of Natural and Environmental Sciences, Newcastle University, Newcastle Upon Tyne NE1 7RU, UK; \\ kirsten.wolff@newcastle.ac.uk \\ 4 Instituto de Biología, Universidad de Antioquia, Medellín 050010, Colombia; lucia.pabon@udea.edu.co \\ 5 Sede Bogotá, Facultad de Ciencias, Instituto de Ciencias Naturales, Universidad Nacional de Colombia, \\ Bogotá 111321, Colombia; fagonzalezg@unal.edu.co \\ * Correspondence: jpmower@unl.edu
}

\section{check for}

updates

Citation: Mower, J.P.; Hanley, L.; Wolff, K.; Pabón-Mora, N.; González, F. Complete Mitogenomes of Two Aragoa Species and Phylogeny of Plantagineae (Plantaginaceae,

Lamiales) Using Mitochondrial Genes and the Nuclear Ribosomal RNA

Repeat. Plants 2021, 10, 2673.

https://doi.org/10.3390/

plants10122673

Academic Editor: Alexey Shipunov

Received: 18 November 2021

Accepted: 2 December 2021

Published: 5 December 2021

Publisher's Note: MDPI stays neutral with regard to jurisdictional claims in published maps and institutional affiliations.

Copyright: (c) 2021 by the authors. Licensee MDPI, Basel, Switzerland. This article is an open access article distributed under the terms and conditions of the Creative Commons Attribution (CC BY) license (https:// creativecommons.org/licenses/by/ $4.0 /)$.
Abstract: Aragoa, comprising 19 high-altitude North Andean species, is one of three genera in the Plantagineae (Plantaginaceae, Lamiales), along with Littorella and Plantago. Based primarily on plastid data and nuclear ITS, Aragoa is sister to a clade of Littorella + Plantago, but Plantagineae relationships have yet to be assessed using multigene datasets from the nuclear and mitochondrial genomes. Here, complete mitogenomes were assembled for two species of Aragoa (A. abietina and A. cleefii). The mitogenomes of both species have a typical suite of genes for 34 proteins, 17 tRNAs, and three rRNAs. The A abietina mitogenome assembled into a simple circular map, with no large repeats capable of producing alternative isoforms. The A. cleefii mitogenomic map was more complex, involving two circular maps bridged by a substoichiometric linear fragment. Phylogenetics of three mitochondrial genes or the nuclear rRNA repeat placed Aragoa as sister to Littorella + Plantago, consistent with previous studies. However, P. nubicola, the sole representative of subg. Bougueria, was nested within subg. Psyllium based on the mitochondrial and nuclear data, conflicting with plastid-based analyses. Phylogenetics of the nuclear rRNA repeat provided better resolution overall, whereas relationships from mitochondrial data were hindered by extensive substitution rate variation among lineages.

Keywords: Aragoa; mitochondrial genome; nuclear rRNA; phylogenetics; Plantagineae

\section{Introduction}

Aragoa is a genus of 19 woody shrubs that reside at high altitudes in the páramos of the Northern Andes of Colombia and Venezuela [1-3]. Based on analyses of nuclear ITS, various plastid genes, or the entire plastid genome [3-8], Aragoa was shown to be sister to Plantago ( 250 species) and Littorella (three species), which altogether make up the Plantagineae [6]. Recently, whole plastome data [8,9] and combined 5-loci analyses from all three plant genomes [10] have shown promise for resolving relationships among Plantagineae and within Plantago. However, sampling of nuclear and mitochondrial loci has been limited to nuclear ITS [3-6,10] and single mitochondrial genes [10,11]. Thus, it is currently unclear whether the plastome results are congruent with the phylogenetic signal in the mitochondrial and nuclear genomes.

To begin to assess the mitogenomic signal, we sequenced and assembled complete mitogenomes for two species of Aragoa (A. abietina and A. cleefii), which represent distinct groups within the genus [1,2]. We augmented these genomic data with published sequences for three mitochondrial genes ( $a t p 1, \operatorname{cox} 1$, and $r r n S)$ that have been widely sequenced from previous Plantaginaceae studies [11,12], and by extracting these mitochondrial genes from Illumina assemblies of 10 additional Plantaginaceae species. In parallel, we performed an 
analysis of the nuclear ribosomal RNA (rRNA) cluster, which includes genes for 18S, 5.8S, and $26 \mathrm{~S}$ rRNAs separated by ITS1 and ITS2 regions. This dataset combined available data from GenBank with extracted nuclear rRNA sequences present in previous assemblies used to capture the plastid genome [8] and from the assemblies performed in this study. Using these data, we describe the complete mitogenomes of two Aragoa species and compare the phylogenetic signal for Plantagineae relationships using mitochondrial and nuclear loci with previous plastid phylogenomic results.

\section{Results}

\subsection{Aragoa Mitogenomic Content and Structure}

The mitochondrial genomes of A. abietina $(365 \mathrm{~kb})$ and A. cleefii $(366 \mathrm{~kb})$ were assembled from an Illumina paired-end library with a large-insert size ( $\sim 800 \mathrm{bp})$. In terms of annotated content, both mitogenomes contain the same set of intact and putatively functional genes encoding 34 proteins, 17 transfer RNAs (tRNAs), and three rRNAs (Figure 1a,b). Repeat content includes the same set of 10 repeats in both species (minimum $100 \mathrm{bp}$ in length and $>90 \%$ sequence identity), the largest of which is only $483 \mathrm{bp}$ in length. Both mitogenomes also contain the same 15 segments of mitochondrial DNA originating from plastid (MIPTs, minimum $100 \mathrm{bp}$ in length and $80 \%$ sequence identity), of which two are $>1 \mathrm{~kb}$ in size at $1.7 \mathrm{~kb}$ and $1.3 \mathrm{~kb}$ (Figure $1 \mathrm{a}, \mathrm{b}$ ).

Read-pairs were mapped to each genome to evaluate depth of sequencing coverage and also to assess the positional consistency of each read pair, which should map in a headto-head orientation at an approximate distance of the library insert size (800 bp $\pm 50 \%$ ). For A. abietina, depth of coverage was consistent across the mitogenome (mean $35.0 \times$, median $34.6 \times$ ), except for two coverage spikes at positions 246 and $259 \mathrm{~kb}$ in the assembly, corresponding to the two largest MIPTs (Figure 1a). Nearly all read pairs mapped in a consistent manner, except that 46 read pairs mapped at the two ends of the scaffold, providing support for a circular mapping assembly (Figure 1c).

For the $A$. cleefii mitogenome, coverage depth was also generally consistent (mean $28.4 \times$, median $28.1 \times$ ), except for two coverage spikes at the two largest MIPTs at positions 324 and $337 \mathrm{~kb}$, and for a substantial dip in coverage (mean and median $=10.9 \times$ ) at a $4.4 \mathrm{~kb}$ region at position 165,852-170,296 (Figure 1b). Read pairs also mapped consistently to the $A$. cleefii mitogenome assembly, except for 57 read pairs that mapped to the left end of the scaffold and the left flank of the low-coverage region, and another 71 read pairs that mapped to the right end of the scaffold and the right flank of the low-coverage region. Thus, the coverage analysis and read-pair consistency mapping support a complex assembly involving two circular maps of 195 and $166 \mathrm{~kb}$ and a substoichiometric linear segment of $4.4 \mathrm{~kb}$ (Figure 1d). Notably, the $195 \mathrm{~kb}$ circular map reconstructs a contiguous rrn26 gene that was split into two distally spaced pieces in the linear arrangement shown in Figure 1b.

No other inconsistently mapping clusters of read pairs (minimum support of five clustered read pairs in a $1 \mathrm{~kb}$ window) were detected in either Aragoa mitogenome, indicating that no other alternative isoforms exist. This finding is consistent with the fact that neither genome has a large repeat $(>1 \mathrm{~kb})$ that might promote high-frequency repeat-mediated rearrangement.

\subsection{Plantagineae Phylogeny Based on Three Mitochondrial Genes}

The phylogram from a phylogenetic analysis of three mitochondrial genes (atp1, cox1, and $r r n 18$ ) indicates substantial rate variation among Plantagineae taxa (Figure 2a), with particularly high levels of sequence divergence for Littorella and Plantago, as described previously $[11,13]$. The cladogram (Figure $2 b$ ) indicates that Plantagineae is weakly supported as monophyletic ( $\mathrm{BS}=58 \%$ ) and sister to Digitalideae (represented by Digitalis) with moderate support (71\%). Within Plantagineae, Aragoa is monophyletic $(100 \%)$ and sister to a clade comprising Littorella and Plantago (100\%), and Littorella is moderately supported as sister to a monophyletic Plantago (69\%). Among Plantago subgenera, the monotypic subg. Bougueria (represented by P. nubicola) is nested within subg. Psyllium with strong support 
(97\%), and a sister relationship between subgenera Coronopus and Plantago is strongly supported $(94 \%)$.

(a)

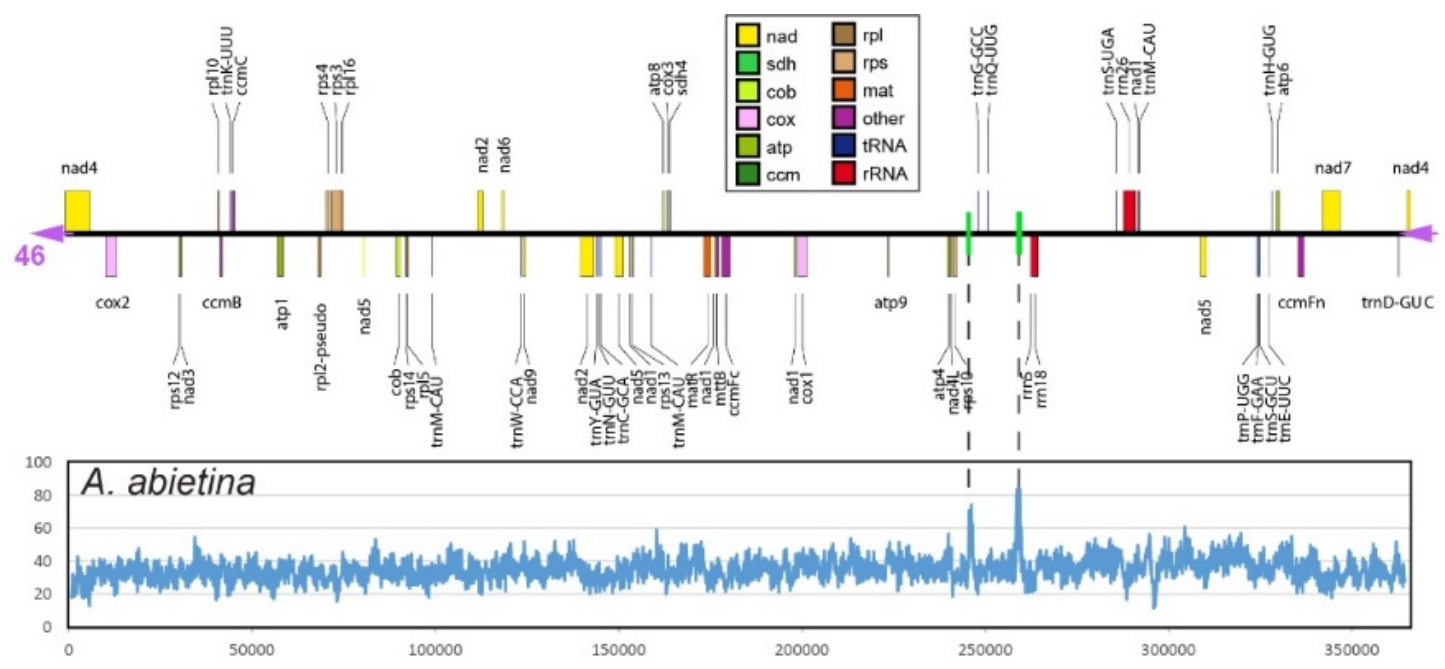

(b)

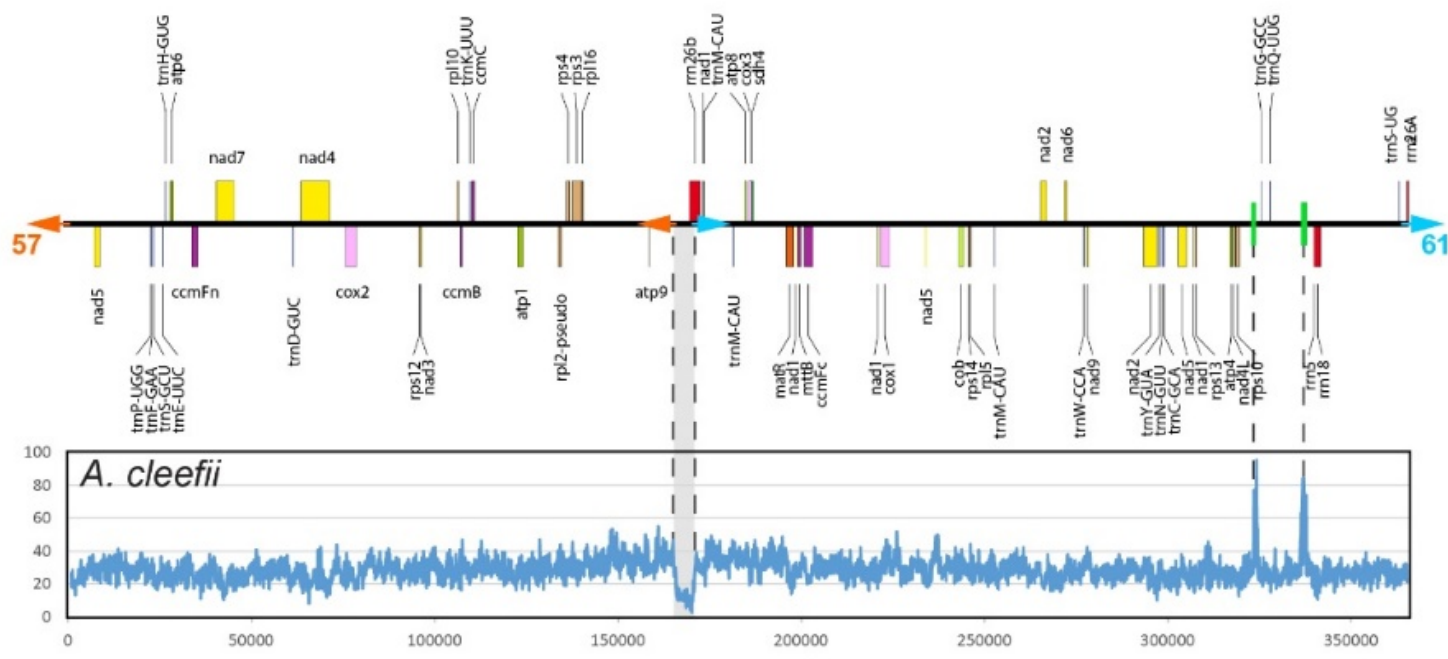

(c)

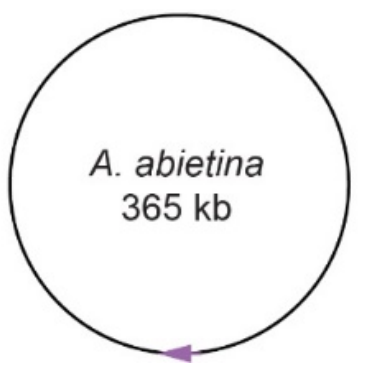

(d)

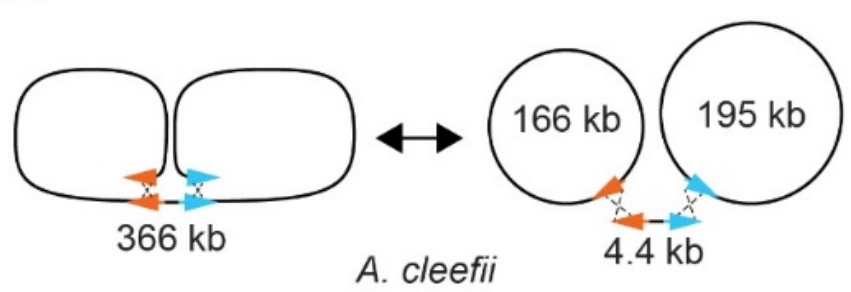

Figure 1. Linear gene map and depth of sequencing coverage for Aragoa mitogenomes. (a) Aragoa abietina. (b) Aragoa cleefii. Genes are color coded according to their functional group as shown in the key at the top. All MIPTs $>500$ bp are shown in green. Positions of atypically high or low coverage are indicated with dotted lines connecting the coverage plot and gene map. Arrowheads indicate structural connections supporting a circularized assembly, and the number of read pairs supporting these connections are indicated. (c) Inferred circularized assembly for the A. abietina mitogenome. (d) Inferred assembly structures for $A$. cleefii, including the putative interconversion of a linearized form and a set of circular and linear forms. Note that these are assembled structures only and may not represent in vivo forms. 
(a)

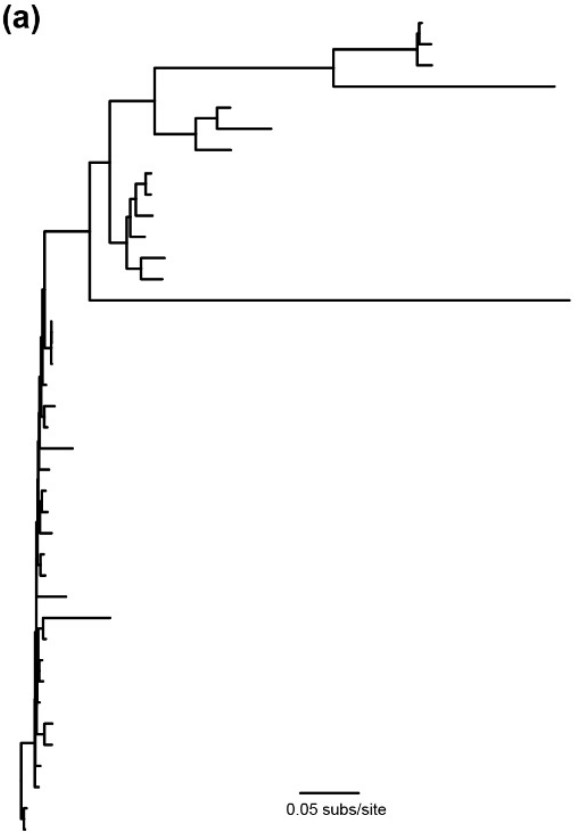

(c)

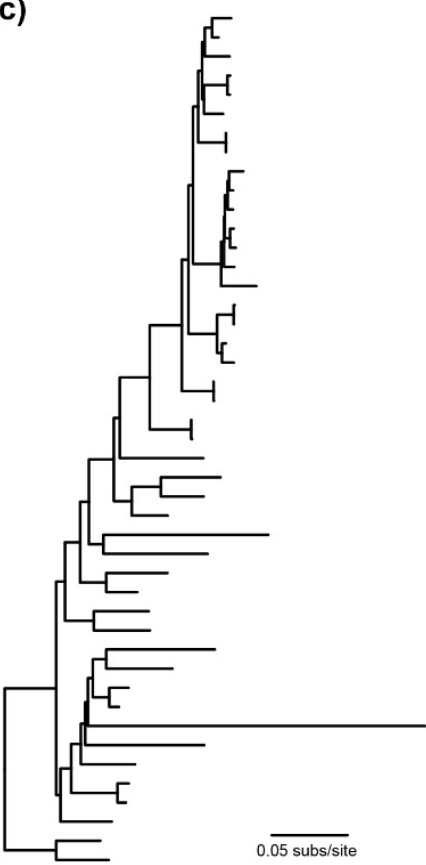

(d) (b)
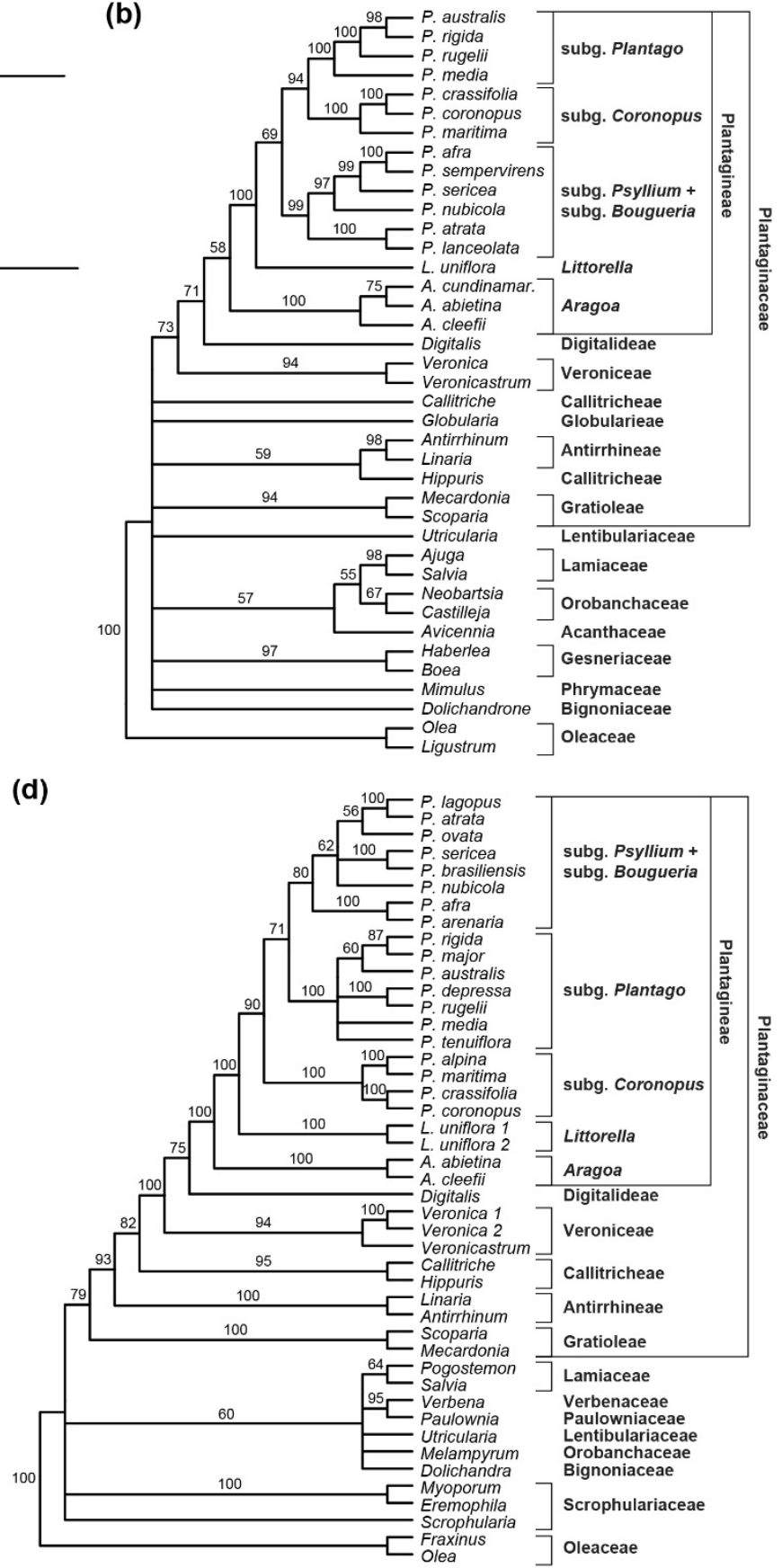

Figure 2. Phylogenetic relationships among Plantaginaceae tribes and Plantagineae genera. (a) Phylogram and (b) cladogram based on three concatenated mitochondrial genes. (c) Phylogram and (d) cladogram based on the nuclear rRNA cluster. Branches with $<50 \%$ bootstrap support were collapsed in the cladograms.

Single-gene phylogenetic analyses of each mitochondrial gene were also performed (Figure S1). All three single-gene trees provide good support for the monophyly of subg. Plantago (BS $=100 \%, 99 \%$, and $100 \%$ for atp1, cox1, and $r r n 18$, respectively) and for the clade comprising Littorella + Plantago (79\%, 99\%, and 95\%). Most other relationships are somewhat inconsistent among trees and received generally poor bootstrap support, suggesting limited phylogenetic signal in the single-gene datasets. 


\subsection{Plantagineae Phylogeny Based on the Nuclear rRNA Repeat}

A phylogram, based on the nuclear rRNA repeat cluster, indicates that this locus exhibits much less rate variation among species, with increased divergence limited to Utricularia (Figure 2c). The cladogram indicates that the bootstrap support is generally more robust for this nuclear dataset (Figure 2d). Consistent with the mitochondrial results, Plantagineae is shown to be monophyletic $(100 \%)$ and sister to Digitalideae $(75 \%)$, and Aragoa and Littorella are successive sisters (100\%, 100\%) to a monophyletic Plantago (90\%). Moreover, $P$. nubicola is again found to be nested within subg. Psyllium, although with limited support (62\%). In contrast to the mitochondrial results, the subg. Bougueria + subg. Psyllium clade associates with subg. Plantago (71\%), placing subg. Coronopus as sister to the other Plantago subgenera with moderate support.

\section{Discussion}

Organellar genomes often assemble into circular maps. However, the predominant mitogenomic structure for many eukaryotes (particularly plants and fungi) is not a circular chromosome but instead a collection of linear molecules of variable size and more complex multibranched linear forms [14,15]. In plants, multiple isoform maps are often inferred due to recombination at large repeats that promote genomic rearrangements [16]. Here, the $A$. abietina mitogenome also mapped as a circular molecule (Figure $1 \mathrm{~b}, \mathrm{~d}$ ). However, the absence of large repeats $(>1 \mathrm{~kb})$ indicates that this mitogenome map has no major alternative isoforms, which was corroborated by the absence of inconsistently mapping read pairs that would indicate a major alternative arrangement. The lack of any large, recombinogenic repeats is uncommon for a plant mitogenome, although some other plants also lack large mitochondrial repeats, such as Brassica hirta [17], Batis maritima [18], Ajuga reptans [19], and 13 of 72 angiosperms evaluated in a broader study [20].

The assembly map for the $A$. cleefii mitogenome was more atypical. The genome assembled into a $366 \mathrm{~kb}$ linear scaffold; however, a $4.4 \mathrm{~kb}$ internal segment was clearly substoichiometric (by about a factor of 3 ) relative to the rest of the assembly (Figure 1b), indicating that this assembled form does not accurately represent the in vivo stoichiometry of the mitogenome. Moreover, no read-pair clusters were found to span the two ends of the $366 \mathrm{~kb}$ linear scaffold, arguing against a single circularized map. Instead, substantial read pairs supported an assembly involving two independent circular maps that are infrequently interconnected by the substoichiometric $4.4 \mathrm{~kb}$ linear segment (Figure 1d). In some sense, the linearization of the $A$. cleefii circular maps by a small linear segment is reminiscent of linear plasmids that linearize the maize CMS-S mitogenome [21,22]. However, the $4.4 \mathrm{~kb}$ linear segment in A. cleefii does not have any terminal inverted repeats or evidence of genes (such as DNA or RNA polymerase) typical of a mitochondrial plasmid [22,23], arguing against the possibility that this $4.4 \mathrm{~kb}$ segment is a mitochondrial plasmid. Similar to A. cleefii, the mitogenome maps for cytoplasmic male-sterile lines of carrot and beet could not be completely circularized, with some fraction of the genome remaining linearized $[24,25]$, suggesting that noncircular maps may be more common among plants than currently recognized. The in vivo structure of the A. cleefii mitogenome requires further investigation.

The nuclear and mitochondrial datasets examined here (Figure 2) represent the largest sets of nuclear and mitochondrial genes for Plantagineae phylogenetics. In general, the results of these analyses are consistent in several respects with previous analyses based on whole plastid genomes [8], single plastid genes, and/or the nuclear ITS [3-5,10], and a combined analysis of five mitochondrial, plastid, and nuclear loci [10]. Collectively, these studies demonstrate that Plantagineae is monophyletic, with Aragoa and Littorella as successive sisters to a monophyletic Plantago. The consistency among studies, and particularly among the three plant genomes, indicates that these conclusions are robust. Moreover, the inferred relationships are unlikely to be influenced by the minor differences in taxon sampling among datasets. The results of this study and the previous plastome-based study [8] also suggest that Plantagineae may be most closely related to Digitalideae, although not 
all Plantaginaceae tribes were sampled in either study. Resolution of relationships among Plantaginaceae tribes will require more extensive sampling of all tribes from all three plant genomes.

Within Plantago, relationships inferred from the mitochondrial and nuclear analyses from this study and the predominantly plastid-based analyses of previous studies were less consistent. For example, plastome data strongly supports a sister-group relationship between subg. Bougueria and subg. Psyllium $[4,8]$, whereas subg. Bougueria is nested within subg. Psyllium based on the mitochondrial and nuclear datasets examined here (Figure 2). In addition, subg. Coronopus is sister to subg. Plantago in the mitochondrial and plastid analyses but sister to the remainder of Plantago based on the nuclear rRNA data. Phylogenetic incongruence among genomes can have a variety of causes, ranging from technical (e.g., limited taxon or locus sampling) to biological (e.g., introgression, incomplete lineage sorting, reticulation). Here, it should be noted that previous whole plastome studies $[8,9]$ analyzed a much larger dataset relative to the mitochondrial and nuclear datasets examined here; additional sampling of nuclear and mitochondrial loci is likely to improve support values for these analyses.

Finally, the extreme rate variation in the mitochondrial dataset suggests that the mitochondrial results should be interpreted with caution. In one sense, the higher mitochondrial substitution rates in Littorella, subg. Coronopus, and subg. Plantago may be beneficial in producing an abundance of variable characters. However, the reliability of the inferred relationships may be negatively affected by issues of long-branch attraction. Given the incongruence among genomes for Plantago relationships, and the huge variation in mitochondrial substitution rates among taxa, we urge caution in combining datasets derived from different genomes for the study of Plantago phylogenetics.

\section{Materials and Methods}

\subsection{Generation of Aragoa Mitochondrial Genomes}

Tissue was collected in Colombia and silica-dried for two species of Aragoa: A. abietina [voucher Natalia Pabón-Mora \& Favio González 288 (COL, HUA)] and A. cleefii [voucher Favio González 4614 (COL, HUA)]. DNA was extracted by use of a CTAB-based protocol [26] and then sequenced by BGI (Shenzhen, China) on an Illumina HiSeq 2500 machine to generate $6.3 \mathrm{~Gb}$ (for A. abietina) or $7.0 \mathrm{~Gb}$ (for A. cleefii) of $2 \times 125$ paired-end sequence data from an 800 bp library (Table S1). Illumina sequence data for both species were assembled with Velvet v1.2.10 [27] and SPAdes v3.11.1 [28], using methods described previously [29]. For each species, mitochondrial contigs were identified using blastn v2.2.31 (with default settings) with a set of mitochondrial reference genes found in angiosperms [30] as queries. A consensus mitogenome sequence was generated by manual alignment of overlapping mitochondrial contigs from the Velvet and SPAdes assemblies.

For each Aragoa mitogenome, protein and rRNA genes were annotated by identifying their position using blastn with the mitochondrial reference genes as queries. When necessary, start and stop codon positions were manually adjusted if their position shifted in Aragoa relative to the reference genes. Genes for tRNAs were identified using tRNAscanSE [31] as implemented in the GeSeq web portal [32].

Depth of coverage was calculated as described previously [33]. Briefly, Illumina readpairs were mapped to the complete genome with blastn v2.2.31, requiring a minimum match of $90 \%$ length and 90\% sequence identity. Read-pairs were classified as "consistent" if they mapped in a head-to-head orientation at a distance consistent with the library insert size ( $800 \mathrm{bp} \pm 50 \%)$. Read-pairs that did not map consistently were classified as "inconsistent" and were binned in $1 \mathrm{~kb}$ windows. Clusters of inconsistent read pairs were identified when a minimum of 5 read pairs mapped to the same pair of $1 \mathrm{~kb}$ windows.

\subsection{Generation of Mitochondrial Gene Sequences}

DNA from Littorella uniflora, Plantago afra, and Plantago nubicola was extracted from fresh or silica-dried material and Illumina sequenced using the same procedures described 
above for Aragoa (Table S1). Illumina or Ion Torrent genome sequencing data were obtained from the NCBI SRA for seven additional Plantagineae species (Table S1). Sequence data for these ten species were assembled with Velvet v1.2.10 and SPAdes v3.11.1 as described above for Aragoa. For each species, contigs containing the mitochondrial genes atp1, cox1, and $r r n 18$ were identified using blastn version 2.2.31 (with default settings), and the sequences of these genes were directly extracted from the contigs. For A. cundinamarcensis, genomic DNA was obtained from the Royal Botanic Gardens (Kew, U.K.) DNA Bank (accession ID 11177), and the atp1 gene was amplified using primers and PCR conditions as described previously [12]; the mitochondrial $r r n 18$ and nuclear rRNA loci were not amplified from this DNA. These newly generated mitochondrial gene sequences were deposited in GenBank, and sequences from additional species were obtained from GenBank (Table S2).

\subsection{Generation of Nuclear Ribosomal RNA Sequences}

During the sequencing and assembly of Plantagineae plastid genomes [8] and/or the draft mitogenomes for this study (Table S1), the nuclear rRNA repeat cluster (including 18S, ITS1, 5.8S, ITS2, 26S) was usually recovered in one or a few contigs. These contigs containing nuclear rRNA sequences were identified using blastn version 2.2.31 (with default settings), and then they were manually aligned based on sequence overlap to build a consensus sequence of the entire nuclear rRNA cluster. The 18S, 5.8S, and 26S rRNA genes were annotated based on comparison to the annotated nuclear rRNA clusters from Eremophila crassifolia and Paulownia coreana (GenBank accessions MN411425 and KP718623, respectively). The newly generated nuclear rRNA sequences were deposited in GenBank and supplemented with additional sequences available in GenBank (Table S3).

\subsection{Phylogenetic Analyses}

Sequences of mitochondrial atp1, cox1, and 18S rRNA genes (Table S2) and the nuclear rRNA repeat cluster (Table S3) were aligned using MUSCLE version 3.8.31 [34]. Ambiguously aligned regions in each dataset were trimmed using Gblocks version 0.91b [35] with relaxed parameter settings $(-t=d-b 1=$ half $-b 2=$ half $-b 3=8-b 4=5-b 5=$ half $)$. A trimmed, concatenated 3-gene mitochondrial dataset was also created using Gblocks in batch mode $(-\mathrm{a}=\mathrm{y})$ with the same relaxed parameter settings. Phylogenetic analyses of each trimmed dataset were performed using RAxML version 8.2.4 [36] with a GTR+G+I model (general time-reversible nucleotide model with gamma-distributed rate variation and a proportion of invariant sites) and 1000 rapid bootstrap replicates.

Supplementary Materials: The following are available online at https:/ / www.mdpi.com/article/10.3 390/plants10122673/s1, Supplementary File S1: File includes Figure S1, Tables S1-S3.

Author Contributions: J.P.M. conceived of the project. J.P.M. and L.H. performed analyses and interpreted results. K.W., N.P.-M., and F.G. contributed resources. J.P.M. wrote the manuscript. All authors have read and agreed to the published version of the manuscript.

Funding: Research was funded in part by the National Science Foundation (award MCB 1125386 to JPM) and the Natural Environment Research Council (award BH111519 to KW).

Data Availability Statement: All newly generated data were deposited in GenBank under accessions OK514181, OK514182, OK523398-OK523436, OK559378-OK559395, and OK959863-OK959867. See Tables S2 and S3 for more details.

Acknowledgments: The authors wish to thank Yizhong Zhang and Nick Levsen for assistance with DNA extractions and sequencing library preparations, Štěpán Husák (Institute of Botany, Czech Academy of Sciences) for providing Littorella uniflora plants, and Stephan Beck (Herbario Nacional de Bolivia) for providing Plantago nubicola tissue.

Conflicts of Interest: The authors declare no conflict of interest. 


\section{References}

1. Fernández Alonso, J.L. Novedades taxonómicas en Aragoa HBK (Scrophulariaceae) y sinopsis del género. An. Jard. Bot. Madrid 1993, 51, 73-96.

2. Fernández Alonso, J.L. Scrophulariaceae-Aragoeae. In Flora de Colombia 16; Instituto de Ciencias Naturales, Universidad Nacional de Colombia: Bogotá, Colombia, 1995.

3. Bello, M.; Chase, M.; Olmstead, R.; Rønsted, N.; Albach, D. The páramo endemic Aragoa is the sister genus of Plantago (Plantaginaceae; Lamiales): Evidence from plastid rbcL and nuclear ribosomal ITS sequence data. Kew Bull. 2002, 57, 585-597. [CrossRef]

4. Rønsted, N.; Chase, M.W.; Albach, D.C.; Bello, M.A. Phylogenetic relationships within Plantago (Plantaginaceae): Evidence from nuclear ribosomal ITS and plastid trnL-F sequence data. Bot. J. Linn. Soc. 2002, 139, 323-338. [CrossRef]

5. Hoggard, R.K.; Kores, P.J.; Molvray, M.; Hoggard, G.D.; Broughton, D.A. Molecular systematics and biogeography of the amphibious genus Littorella (Plantaginaceae). Am. J. Bot. 2003, 90, 429-435. [CrossRef]

6. Albach, D.C.; Meudt, H.M.; Oxelman, B. Piecing together the "new" Plantaginaceae. Am. J. Bot. 2005, 92, 297-315. [CrossRef]

7. Affenzeller, M.; Kadereit, J.W.; Comes, H.P. Parallel bursts of recent and rapid radiation in the Mediterranean and Eritreo-Arabian biodiversity hotspots as revealed by Globularia and Campylanthus (Plantaginaceae). J. Biogeogr. 2018, 45, 552-566. [CrossRef]

8. Mower, J.P.; Guo, W.; Partha, R.; Fan, W.; Levsen, N.; Wolff, K.; Nugent, J.M.; Pabon-Mora, N.; Gonzalez, F. Plastomes from tribe Plantagineae (Plantaginaceae) reveal infrageneric structural synapormorphies and localized hypermutation for Plantago and functional loss of $n d h$ genes from Littorella. Mol. Phylogenet. Evol. 2021, 162, 107217. [CrossRef]

9. Hassemer, G.; Bruun-Lund, S.; Shipunov, A.B.; Briggs, B.G.; Meudt, H.M.; Ronsted, N. The application of high-throughput sequencing for taxonomy: The case of Plantago subg. Plantago (Plantaginaceae). Mol. Phylogenet. Evol. 2019, 138, 156-173. [CrossRef] [PubMed]

10. Shipunov, A.; Fernández-Alonso, J.L.; Hassemer, G.; Alp, S.; Lee, H.J.; Pay, K. Molecular and morphological data Improve the classification of Plantagineae (Lamiales). Plants 2021, 10, 2299. [CrossRef]

11. Cho, Y.; Mower, J.P.; Qiu, Y.L.; Palmer, J.D. Mitochondrial substitution rates are extraordinarily elevated and variable in a genus of flowering plants. Proc. Natl. Acad. Sci. USA 2004, 101, 17741-17746. [CrossRef] [PubMed]

12. Mower, J.P.; Stefanović, S.; Hao, W.; Gummow, J.S.; Jain, K.; Ahmed, D.; Palmer, J.D. Horizontal acquisition of multiple mitochondrial genes from a parasitic plant followed by gene conversion with host mitochondrial genes. BMC Biol. 2010, 8,150 [CrossRef]

13. Mower, J.P.; Touzet, P.; Gummow, J.S.; Delph, L.F.; Palmer, J.D. Extensive variation in synonymous substitution rates in mitochondrial genes of seed plants. BMC Evol. Biol. 2007, 7, 135. [CrossRef]

14. Bendich, A.J. Reaching for the ring: The study of mitochondrial genome structure. Curr. Genet. 1993, 24, 279-290. [CrossRef]

15. Smith, D.R.; Keeling, P.J. Mitochondrial and plastid genome architecture: Reoccurring themes, but significant differences at the extremes. Proc. Natl. Acad. Sci. USA 2015, 112, 10177-10184. [CrossRef]

16. Gualberto, J.M.; Newton, K.J. Plant mitochondrial genomes: Dynamics and mechanisms of mutation. Annu. Rev. Plant Biol. 2017, 68, 225-252. [CrossRef] [PubMed]

17. Palmer, J.D.; Herbon, L.A. Unicircular structure of the Brassica hirta mitochondrial genome. Curr. Genet. 1987, 11, 565-570. [CrossRef] [PubMed]

18. Grewe, F.; Edger, P.P.; Keren, I.; Sultan, L.; Pires, J.C.; Ostersetzer-Biran, O.; Mower, J.P. Comparative analysis of 11 Brassicales mitochondrial genomes and the mitochondrial transcriptome of Brassica oleracea. Mitochondrion 2014, 19, 135-143. [CrossRef] [PubMed]

19. Zhu, A.; Guo, W.; Jain, K.; Mower, J.P. Unprecedented heterogeneity in the synonymous substitution rate within a plant genome. Mol. Biol. Evol. 2014, 31, 1228-1236. [CrossRef] [PubMed]

20. Wynn, E.L.; Christensen, A.C. Repeats of unusual size in plant mitochondrial genomes: Identification, incidence and evolution. G3-Genes Genom. Genet. 2019, 9, 549-559. [CrossRef] [PubMed]

21. Schardl, C.L.; Lonsdale, D.M.; Pring, D.R.; Rose, K.R. Linearization of maize mitochondrial chromosomes by recombination with linear episomes. Nature 1984, 310, 292-296. [CrossRef]

22. Allen, J.O.; Fauron, C.M.; Minx, P.; Roark, L.; Oddiraju, S.; Lin, G.N.; Meyer, L.; Sun, H.; Kim, K.; Wang, C.; et al. Comparisons among two fertile and three male-sterile mitochondrial genomes of maize. Genetics 2007, 177, 1173-1192. [CrossRef]

23. Handa, H. The complete nucleotide sequence and RNA editing content of the mitochondrial genome of rapeseed (Brassica napus L.): Comparative analysis of the mitochondrial genomes of rapeseed and Arabidopsis thaliana. Nucleic Acids Res. 2003, 31, 5907-5916. [CrossRef] [PubMed]

24. Kubo, T.; Nishizawa, S.; Mikami, T. Alterations in organization and transcription of the mitochondrial genome of cytoplasmic male sterile sugar beet (Beta vulgaris L.). Mol. Gen. Genet. 1999, 262, 283-290. [CrossRef]

25. Robison, M.; Wolyn, D. Complex organization of the mitochondrial genome of petaloid CMS carrot. Mol. Genet. Genom. 2002, 268, 232-239. [CrossRef] [PubMed]

26. Doyle, J.J.; Doyle, J.L. A rapid DNA isolation procedure for small quantities of fresh leaf tissue. Phytochem. Bull. 1987, 19, 11-15.

27. Zerbino, D.R.; Birney, E. Velvet: Algorithms for de novo short read assembly using de Bruijn graphs. Genome Res. 2008, 18, 821-829. [CrossRef] 
28. Bankevich, A.; Nurk, S.; Antipov, D.; Gurevich, A.A.; Dvorkin, M.; Kulikov, A.S.; Lesin, V.M.; Nikolenko, S.I.; Pham, S.; Prjibelski, A.D.; et al. SPAdes: A new genome assembly algorithm and its applications to single-cell sequencing. J. Comput. Biol. 2012, 19, 455-477. [CrossRef]

29. Mower, J.P.; Ma, P.F.; Grewe, F.; Taylor, A.; Michael, T.P.; Vanburen, R.; Qiu, Y.L. Lycophyte plastid genomics: Extreme variation in GC, gene and intron content and multiple inversions between a direct and inverted orientation of the rRNA repeat. New Phytol. 2019, 222, 1061-1075. [CrossRef]

30. Mower, J.P. Variation in protein gene and intron content among land plant mitogenomes. Mitochondrion 2020, 53, 203-213. [CrossRef]

31. Lowe, T.M.; Eddy, S.R. tRNAscan-SE: A program for improved detection of transfer RNA genes in genomic sequence. Nucleic Acids Res. 1997, 25, 955-964. [CrossRef] [PubMed]

32. Tillich, M.; Lehwark, P.; Pellizzer, T.; Ulbricht-Jones, E.S.; Fischer, A.; Bock, R.; Greiner, S. GeSeq-Versatile and accurate annotation of organelle genomes. Nucleic Acids Res. 2017, 45, W6-W11. [CrossRef] [PubMed]

33. Mower, J.P.; Case, A.L.; Floro, E.R.; Willis, J.H. Evidence against equimolarity of large repeat arrangements and a predominant master circle structure of the mitochondrial genome from a monkeyflower (Mimulus guttatus) lineage with cryptic CMS. Genome Biol. Evol. 2012, 4, 670-686. [CrossRef] [PubMed]

34. Edgar, R.C. MUSCLE: Multiple sequence alignment with high accuracy and high throughput. Nucleic Acids Res. 2004, 32, 1792-1797. [CrossRef]

35. Castresana, J. Selection of conserved blocks from multiple alignments for their use in phylogenetic analysis. Mol. Biol. Evol. 2000, 17, 540-552. [CrossRef] [PubMed]

36. Stamatakis, A. RAxML version 8: A tool for phylogenetic analysis and post-analysis of large phylogenies. Bioinformatics 2014, 30, 1312-1313. [CrossRef] [PubMed] 